\title{
INTEGRAL observations of the GeV blazar PKS 1502+106 and the hard X-ray bright Seyfert galaxy Mkn $841^{\star}$
}

\author{
E. Pian ${ }^{1,2,3}$, P. Ubertini ${ }^{4}$, A. Bazzano ${ }^{4}$, V. Beckmann ${ }^{5}$, D. Eckert ${ }^{6}$, G. Ghisellini ${ }^{7}$, T. Pursimo ${ }^{8}$, G. Tagliaferri ${ }^{7}$, \\ F. Tavecchio ${ }^{7}$, M. Türler ${ }^{9}$, S. Bianchi ${ }^{10}$, V. Bianchin ${ }^{11}$, R. Hudec ${ }^{12,13}$, L. Maraschi ${ }^{7}$, C. M. Raiteri ${ }^{14}$, S. Soldi $^{15}$, \\ A. Treves ${ }^{16}$, and M. Villata ${ }^{14}$ \\ 1 INAF, Trieste Astronomical Observatory, via G.B. Tiepolo 11, 34143 Trieste, Italy \\ e-mail: elena.pian@sns.it \\ 2 Scuola Normale Superiore, Piazza dei Cavalieri 7, 56126 Pisa, Italy \\ 3 European Southern Observatory, Karl-Schwarzschild-Strasse 2, 85748 Garching bei München, Germany \\ 4 INAF, IASF-Roma, via del Fosso del Cavaliere, 100, 00133 Roma, Italy \\ 5 Centre François Arago, APC, Université Paris Diderot, CNRS/IN2P3, 10 rue Alice Domon et Léonie Duquet, \\ 75205 Paris Cedex 13, France \\ 6 INAF/IASF Milano, via E. Bassini 15, 20133 Milano, Italy \\ 7 INAF, Brera Astronomical Observatory, via E. Bianchi 46, 23807 Merate (LC), Italy \\ 8 Nordic Optical Telescope, Apartado 474, 38700 Santa Cruz de la Palma, Spain \\ 9 INTEGRAL Science Data Centre, University of Geneva, Chemin d'Ecogia 16, 1290 Versoix, Switzerland \\ 10 Department of Physics, University "Roma Tre", via della Vasca Navale 84, 00146 Roma, Italy \\ 11 INAF, IASF-Bologna, via P. Gobetti, 40129 Bologna, Italy \\ 12 Astronomical Institute, Academy of Sciences, Fricova 298, 25165 Ondrejov, Czech Republic \\ 13 Czech Technical University in Prague, Faculty of Electrical Engineering, Czech Republic \\ 14 INAF, Turin Astronomical Observatory, via Osservatorio 20, 10025 Pino Torinese (TO), Italy \\ 15 Laboratoire AIM - CNRS - CEA/DSM - Université Paris Diderot (UMR 7158), CEA Saclay, DSM/IRFU/SAp, \\ 91191 Gif-sur-Yvette, France \\ 16 Department of Physics and Mathematics, University of Insubria, via Valleggio 11, 22100 Como, Italy
}

Received 16 July 2010 / Accepted 7 October 2010

\begin{abstract}
Context. Extragalactic nuclear activity is most effectively explored with observations at high energies, where the most extreme flux and spectral variations are expected to occur, because of changes in either the accretion flow or the kinematics of the plasma. In active galactic nuclei of blazar type, these variations are the most dramatic.

Aims. By following blazar outbursts from their onset and correlating the observed variations at many different wavelengths, we can reconstruct the behavior of the plasma and map out the development of the flare within the jet.

Methods. The advent of the Fermi satellite has allowed the start of a systematic and intensive monitoring program of blazars. Blazar outbursts are very effectively detected by the LAT instrument in the $\mathrm{MeV}-\mathrm{GeV}$ domain, and these can be promptly followed up with other facilities. Based on a Fermi LAT detection of a high MeV-GeV state, we observed the blazar PKS $1502+106$ with the INTEGRAL satellite between 9 and 11 August 2008. Simultaneous Swift observations were also accomplished, as well as optical follow-up at the Nordic Optical Telescope.

Results. The IBIS instrument onboard INTEGRAL detected a source at a position inconsistent with the optical coordinates of PKS 1502+106, but consistent with those of the Seyfert 1 galaxy Mkn 841, located at 6.8 arcmin south-west of the blazar, which is therefore responsible for all the hard X-ray flux detected by IBIS. At the location of the blazar, IBIS sets an upper limit of $\sim 10^{-11} \mathrm{erg} \mathrm{s}^{-1} \mathrm{~cm}^{-2}$ on the $15-60 \mathrm{keV}$ flux, which is consistent with a model of inverse Compton scattering accounting for the soft X-ray and gamma-ray spectra measured by Swift XRT and Fermi LAT, respectively. The gamma-ray spectrum during the outburst indicates substantial variability in the characteristic energy of the inverse Compton component in this blazar. The hard X-ray state of the Seyfert appears to be nearly unchanged with respect to the past. On the other hand, its soft X-ray flux $(0.3-10 \mathrm{keV})$ varies with a $\sim 50 \%$ amplitude on timescales from days to years. On long timescales, this is well correlated with the optical flux, with no measurable delay.

Conclusions. In PKS $1502+106$, the critical parameters that control variability are the accelerating power transferred to the relativistic electrons, and the magnetic field in the emitting region. The spectrum of Mkn 841 in the $0.3-100 \mathrm{keV}$ range is well described by a power-law with a cutoff at $\sim 150 \mathrm{keV}$ and a Compton reflected continuum.
\end{abstract}

Key words. galaxies: active - quasars: individual: PKS 1502+106 - galaxies: Seyfert - galaxies: individual: Mkn 841 - radiation mechanisms: general - gamma-rays: galaxies

\footnotetext{
* Based on observations made with: INTEGRAL, an ESA project with instruments and Science Data Centre funded by ESA member states (especially the PI countries: Denmark, France, Germany, Italy, Switzerland, Spain), Czech Republic and Poland, and with the
}

participation of Russia and the USA, and the Nordic Optical Telescope, operated on the island of La Palma jointly by Denmark, Finland, Iceland, Norway, and Sweden, in the Spanish Observatorio del Roque de los Muchachos of the Instituto de Astrofisica de Canarias. 


\section{Introduction}

The daily survey of the MeV-GeV sky by the Large Area Telescope (LAT) instrument (Atwood et al. 2009) onboard the Fermi gamma-ray satellite has dramatically enhanced the detection rate of flaring blazars, and will allow an accurate determination of their variability duty cycle at the highest energies. The follow-up of these high states at lower energies and the comparison with quiescent states make it possible to isolate the basic parameters controlling the variations at all frequencies and ultimately place constraints on the behavior of the central engine (Ghisellini et al. 2009; Marscher et al. 2010; Abdo et al. 2010a; Böttcher \& Dermer 2010).

PKS $1502+106(z=1.839$, see Abdo et al. 2010b, for a discussion on this preferred redshift value) is a bright, variable, and optically polarized blazar (Impey \& Tapia 1988; George et al. 1994; Lahteenmaki \& Valtaoja 1999; Lister \& Homan 2005). An et al. (2004) suggested, based on the detection of remarkable superluminal motion with high resolution radio observations, that it should be a strong gamma-ray emitter. The source is not included in the Third EGRET Catalog (Hartman et al. 1999), but was detected in the first three months of sky-survey operation with the LAT (Abdo et al. 2009) and then again with the same instrument during a flaring state on 6 August 2008 (Abdo et al. $2010 \mathrm{~b}$ ) with a flux above $2 \times 10^{-6}$ photons $\mathrm{cm}^{-2} \mathrm{~s}^{-1}$ at energies higher than $100 \mathrm{MeV}$. In January 2009, the source underwent another flare reaching a comparable gamma-ray flux level (Abdo et al. 2010b). It is one of the brightest, most intrinsically luminous, and violently variable AGNs of the Fermi LAT sample (Abdo et al. 2010c,d).

The field of PKS 1502+106 was observed on 24-26 January 2006 by INTEGRAL IBIS for a total integration time of $83 \mathrm{ks}$. The observation, analyzed by Ubertini et al. (2008) in the light of the Fermi report, resulted in the detection of a new source, IGR J15039+1022, at a flux level of $1.6 \mathrm{mCrab}$ (i.e., $2.45 \times$ $10^{-11} \mathrm{erg} \mathrm{cm}^{-2} \mathrm{~s}^{-1}$ in the $18-60 \mathrm{keV}$ range $\left.^{1}\right)$.

The IBIS source, located at RA $=15: 03: 53.76$ and $\mathrm{Dec}=$ $+10: 22: 08.4$ (error radius of $\sim 5$ arcmin), was tentatively identified with the Seyfert 1 galaxy Mkn $841(z=0.036)$ and included in the fourth INTEGRAL IBIS/ISGRI soft gamma-ray survey catalog (Bird et al. 2010) and the second INTEGRAL AGN catalog (Beckmann et al. 2009). Ubertini et al. (2008) note that PKS $1502+106$ is 11 arcmin away from IGR J15039+1022 and is not detected in the January 2006 IBIS observation. They derive a 2- $\sigma$ upper limit flux of $0.7 \mathrm{mCrab}$ for the blazar in the range 18-60 keV, assuming a Crab-like spectrum.

Prompted by the Fermi detection of the gamma-ray outburst, we activated INTEGRAL observations starting on 9 August 2008 (see preliminary images in Blazek \& Hudec 2009). Simultaneous observations with Swift XRT and the Nordic Optical Telescope (NOT) were also organized. We report here the results and discuss them in the context of AGN models.

\section{Observations and data analysis}

\subsection{INTEGRAL}

The source PKS 1502+106 (whose catalogued optical J2000 coordinates are RA $=15: 04: 24.98$, Dec $=+10: 29: 39.2$ ) was observed by the INTEGRAL satellite (Winkler et al. 2003) for 203 ks in Revolution 711 between 2008 August 09, 01:53 UT and 2008 August 11, 15:12 UT. The on-source time for

\footnotetext{
1 Note that this flux supersedes the value of $1.2 \times 10^{-11} \mathrm{erg} \mathrm{cm}^{-2} \mathrm{~s}^{-1}$ reported previously (Ubertini et al. 2008; Abdo et al. 2010b).
}

IBIS/ISGRI (Ubertini et al. 2003; Lebrun et al. 2003) was $194.5 \mathrm{ks}$. The target is in the JEM-X (Lund et al. 2003) field of view for $46 \mathrm{ks}$ in Rev. 711.

The screening, reduction, and analysis of the INTEGRAL IBIS/ISGRI and JEM-X data were performed using the INTEGRAL Offline Scientific Analysis (OSA) v. 7.0 and v. 8.0, respectively, publicly available through the INTEGRAL Science Data Center $^{2}$ (ISDC, Courvoisier et al. 2003). The IBIS/ISGRI and JEM-X data were combined into final images with algorithms implemented in software described in Goldwurm et al. (2003) and Westergaard et al. (2003), respectively. Following similar methods, we also re-analyzed the archival INTEGRAL IBIS and JEM-X observation obtained on 24-26 January 2006 (Ubertini et al. 2008).

The source was also observed with the INTEGRAL OMC (Mas-Hesse et al. 2003) with a standard $V$-band Johnson filter. The data were extracted with default settings, using a $3 \times 3$ pixel binning, which is appropriate for point-like sources.

\subsection{Swift}

X-ray and optical/UV observations of the blazar field were accomplished from 7 to 22 August 2008 with Swift XRT and UVOT (Gehrels et al. 2004; Burrows et al. 2005; Roming et al. 2005), respectively. The pointing included the location of the Seyfert $1 \mathrm{Mkn} 841$. The results of the analysis of the data of PKS $1502+106$ were reported by Abdo et al. (2010b). Following similar procedures as those adopted by those authors, we independently reduced the XRT and UVOT data of the blazar and the Seyfert 1. As in Abdo et al. (2010b), for the blazar, given its low count rate $\left(<0.5\right.$ counts $\left.\mathrm{s}^{-1}\right)$, only photon counting data were considered and XRT grades $0-12$ were selected. No pileup correction was required. For the Seyfert, we also considered window timing mode data. The source events were extracted in circular regions centered on the sources with radii depending on the source intensity (10-20 pixels, 1 pixel $\sim 2$ '. 37), while background events were extracted in source-free annular regions around the sources.

XRT spectra were extracted for each of the two sources from the observation of 8 August 2008, which is the one with the longest exposure time $(12.5 \mathrm{ks})$. Ancillary response files were generated with XRTMKARF and account for different extraction regions, vignetting, and point-spread function corrections. We used the v010 spectral redistribution matrices in the calibration database maintained by HEASARC. All spectra were rebinned with a minimum of 20 counts per energy bin to allow $\chi^{2}$ fitting within XSPEC (v11.3.2). For the spectral fitting, we fixed the hydrogen column density to the Galactic value of $2.22 \times 10^{20} \mathrm{~cm}^{-2}$ (Kalberla et al. 2005).

We extracted XRT average count rates from the same image regions as for the spectra for source and background in the band $0.2-10 \mathrm{keV}$. These light curves were corrected for point-spread function losses, i.e., losses due to the extraction region geometry, vignetting, and bad/hot pixels and columns falling within this region.

Likewise, the UVOT data of both sources were reduced and analyzed following procedures similar to those used by Abdo et al. (2010b). The photometry of Mkn 841 may include partial contamination from the host galaxy bulge. We used an aperture of $6^{\prime \prime}$ for the v,b,u filter images and $12^{\prime \prime}$ for the UV filters. We also reduced and analyzed the Mkn 841 data of 3 February 2007,

\footnotetext{
${ }^{2}$ http://www.isdc. unige.ch/INTEGRAL/download/osa_Sw
} 
Table 1. Log of optical observations at the Nordic Optical Telescope.

\begin{tabular}{cccc}
\hline \hline \multicolumn{1}{c}{ UT $^{a}$} & $T_{\text {expo }}(\mathrm{s})$ & Filter & Magnitude $^{b}$ \\
\hline PKS 1502+106 & \\
\hline 2008 Aug. 13, 21:01:28.0 & 120 & $B_{\text {Bes }}$ & $17.45 \pm 0.09$ \\
2008 Aug. 13, 21:05:33.0 & 80 & $V_{\text {Bes }}$ & $17.05 \pm 0.02$ \\
2008 Aug. 13, 21:08:51.0 & 60 & $R_{\text {Bes }}$ & $16.63 \pm 0.02$ \\
2008 Aug. 13, 21:11:50.0 & 60 & $i_{\text {int }}$ & $16.06 \pm 0.01$ \\
\hline \multicolumn{5}{c}{ Mkn 841 } \\
\hline 2008 Aug. 13, 20:47:22.0 & 20 & $B_{\text {Bes }}$ & $14.90 \pm 0.12$ \\
2008 Aug. 13, 20:50:43.5 & 15 & $V_{\text {Bes }}$ & $14.63 \pm 0.05$ \\
2008 Aug. 13, 20:52:35.0 & 10 & $R_{\text {Bes }}$ & $14.34 \pm 0.03$ \\
2008 Aug. 13, 20:54:41.0 & 10 & $i_{\text {int }}$ & $14.21 \pm 0.04$ \\
\hline
\end{tabular}

Notes. ${ }^{(a)}$ Mid time of observation. ${ }^{(b)}$ Not corrected for Galactic dust absorption. Errors are standard deviations in the photometric zeropoint.

which were included in the Seyfert UVOT study of Grupe et al. (2010).

\subsection{Nordic Optical Telescope}

We observed the IBIS field on 13 August 2008 with the ALFOSC camera and optical BVRI filters at the NOT (Canary Islands). The data were reduced following standard procedures within the IRAF package (overscan subtraction, bias correction, twilight flat fielding). Aperture photometry was performed with the IRAF routine APPHOT. Eight field stars were used to estimate the photometric zeropoint and their magnitudes were taken from the SDSS DR7 (using CDS/Aladin). The field stars SDSS gri- magnitudes were converted to $B V R I$ bands following the transformations ${ }^{3}$ recommended for the SDSS Data Release 5. We refer to Table 1 for a $\log$ of the observations.

\section{Results}

\subsection{INTEGRAL}

A source is detected in hard X-rays with IBIS/ISGRI at coordinates RA $=15: 03: 53.5$, Dec $=+10: 26: 45$, with an error of 2.4 arcmin at $90 \%$ confidence level (see Fig. 1). The detection has an 8.6- $\sigma$ significance in the co-added image of the full observation. The centroid of the error circle of this source is located 8.3 arcmin from the optical source corresponding to PKS 1502+106, thus is inconsistent with the blazar at a 4- $\sigma$ significance level (i.e., the association probability is $\sim 10^{-3}$ ).

However, it is located at only 1.9 arcmin from Mkn 841 (optical catalogue coordinates J2000: $\mathrm{RA}=15: 04: 01.20$, Dec $=$ $+10: 26: 16.2$ ), and therefore consistent with it (see Fig. 1). The error circle of the IBIS detection of January 2006 is also reported in Fig. 1: as already noted, this hard X-ray source is also consistent only with the Seyfert galaxy.

From the count rates measured in our IBIS observation, we derive a flux of $(2.4 \pm 0.3) \times 10^{-11} \mathrm{erg} \mathrm{s}^{-1} \mathrm{~cm}^{-2}$ in the range 15-60 keV for Mkn 841, assuming the spectrum measured by BeppoSAX $(\Gamma=2$, Petrucci et al. 2002; the limited signalto-noise ratio does not allow us to extract a spectrum from the present IBIS observation), and a 3- $\sigma$ upper limit of $9.3 \times$ $10^{-12} \mathrm{erg} \mathrm{s}^{-1} \mathrm{~cm}^{-2}$ in the same energy range for the blazar. These values are similar to the ones determined by Ubertini et al. (2008) from the January 2006 IBIS observation, taking into account the slight difference in energy range.

\footnotetext{
3 http://wWw.sdss.org/DR5/algorithms/ sdssUBVRITransform. html
}

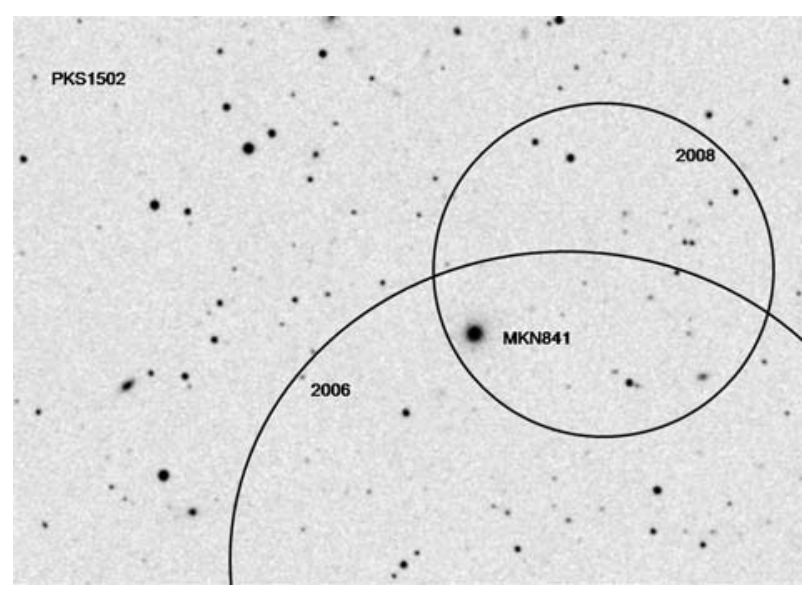

Fig. 1. Digitized Sky Survey image of the field of PKS $1502+106$ and Mkn 841 in red filter. The image vertical size corresponds to 7 arcmin. The two optical sources are indicated. Over-plotted are the error circles of the IBIS detections of January 2006 (larger circle, with a radius of 5 arcmin) and August 2008 (smaller circle, radius of 2.4 arcmin).

Neither PKS 1502+106 nor Mkn 841 are detected by JEM$\mathrm{X}$ in both 2006 and 2008. Assuming a Crab-like spectrum, we estimate 3- $\sigma$ upper limits on the 3-10 keV flux of both sources of $3.3 \times 10^{-11} \mathrm{erg} \mathrm{s}^{-1} \mathrm{~cm}^{-2}$ and $2.3 \times 10^{-11} \mathrm{erg} \mathrm{s}^{-1} \mathrm{~cm}^{-2}$ for 2006 and 2008, respectively (consistent with the XRT measurements, see below).

The OMC measured an optical magnitude of $V=15.4 \pm 0.4$ for PKS $1502+106$. Since this is substantially brighter than the $V$-band flux measured by NOT (see Table 1) and considering the relatively large OMC pixel size (about 18 arcsec), we have checked for possible contamination from nearby sources. Four USNO stars are located within 1 arcmin of the blazar: the summed contribution of their magnitudes, when added to the flux of the blazar measured with the NOT, is comparable to the measured magnitude so they significantly contaminate the blazar light. Therefore, we ignore its OMC optical measurement. The OMC field of view also included Mkn 841, for which we measured an average magnitude of $V=14.33 \pm 0.02$ with no significant variability. No nearby source significantly contaminates the Seyfert optical emission.

\subsection{Fermi LAT}

We re-analyzed the Fermi LAT spectrum acquired for the outburst state presented by Abdo et al. (2010b) using two different time intervals for signal integration: for the same period of five days between 5 and 10 August 2008 adopted by Abdo et al. (2010b), we obtain a photon index of $\Gamma=2.05 \pm 0.04$, consistent with that measured by those authors $(\Gamma=2.06 \pm 0.02)$. For the day of the gamma-ray flare peak, 8 August 2008, we find a photon index of $\Gamma=1.88 \pm 0.08$. These indices are reported in Fig. 3 (see Discussion).

\subsection{Swift}

Comparison of our results with those of Abdo et al. (2010b) for PKS 1502+106 and Grupe et al. (2010) for Mkn 841 shows a general consistency. Swift XRT and UVOT light curves for PKS 1502+106 in August 2008 were reported by Abdo et al. (2010b), who found a decreasing trend in the X-ray flux, the aftermath of the gamma-ray outburst, and a fully resolved flare in optical/UV, that was correlated with the gamma-ray event. In Fig. 2a, we show the light curves of Mkn 841 during the present campaign and in Fig. 2b we report all XRT and UVOT $V$-band 

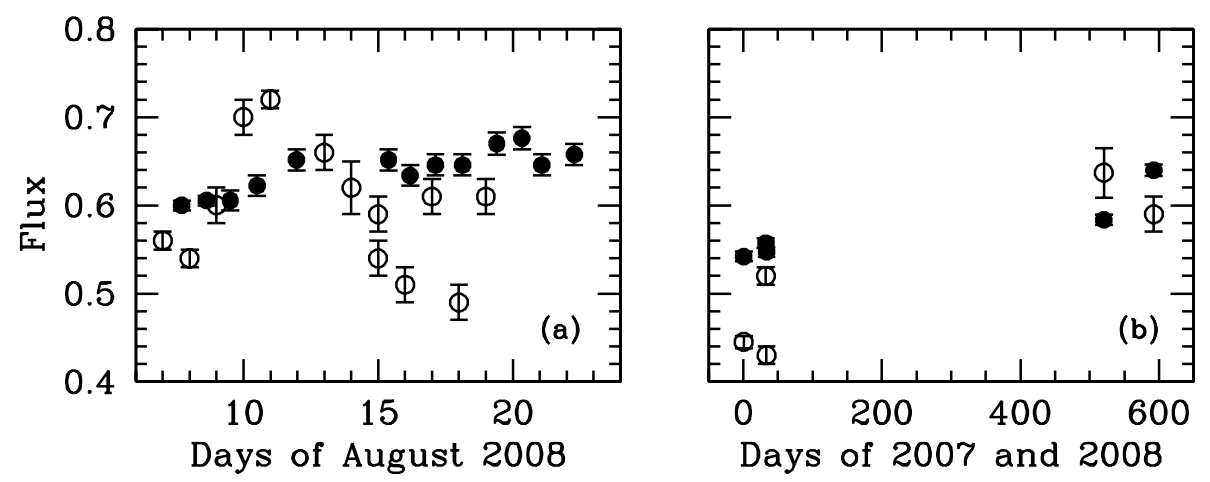

Fig. 2. Swift light curves of Mkn 841: a) in August 2008 and b) in 2007-2008: XRT count rates (in $\mathrm{c} \mathrm{s}^{-1}$, open circles) and UVOT fluxes (filled circles) obtained with an arbitrary magnitude-to-flux conversion coefficient from the $V$-band magnitudes. The errors are statistical only and correspond to $1-\sigma$. The data points in b) are from Grupe et al. (2010), except the last XRT and UVOT ones, which correspond to the averages of the August 2008 points for the two instruments shown in a). The zero time in b) corresponds to 2007 January 1, 00 UT.

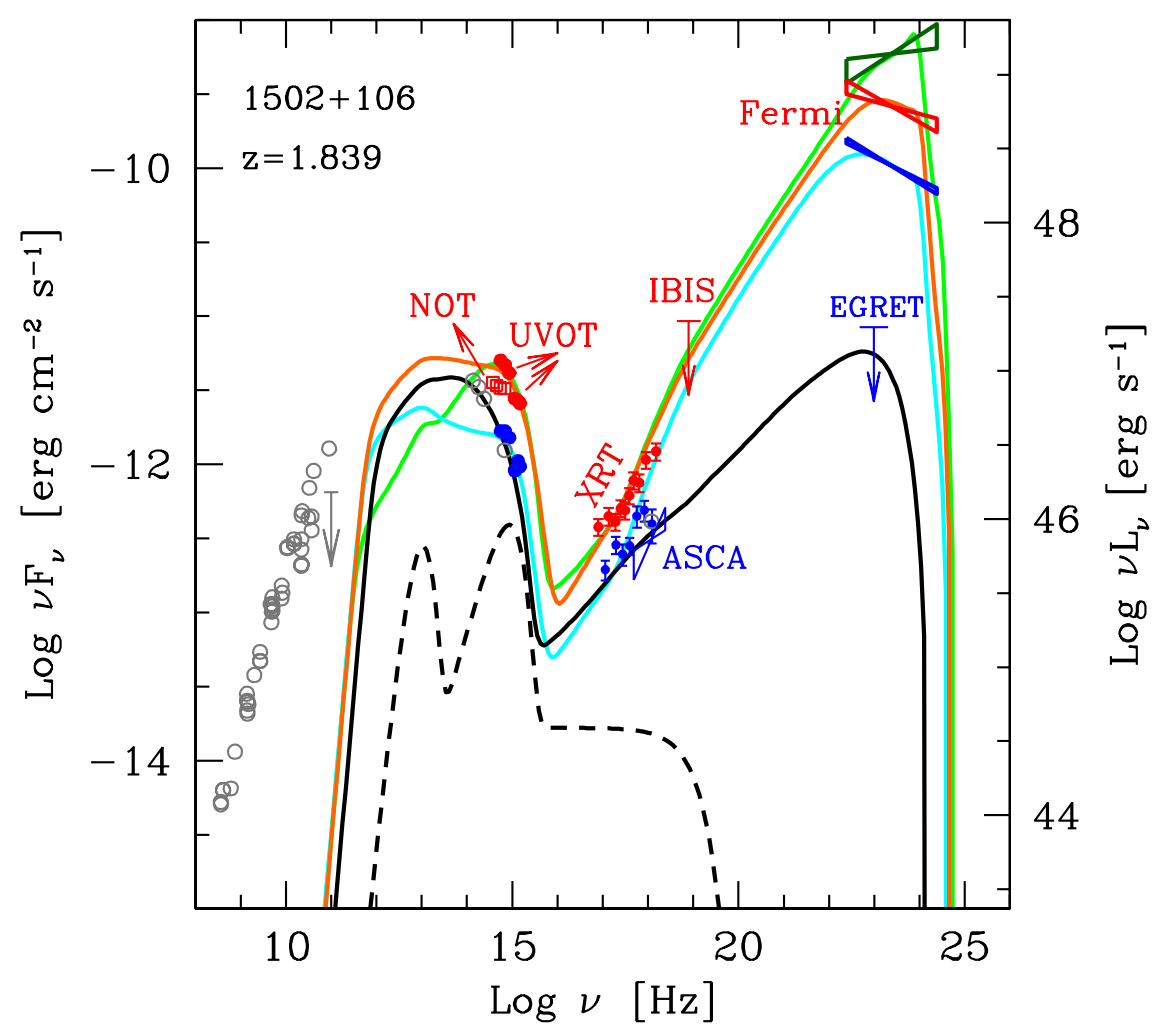

Fig. 3. Spectral energy distributions of PKS $1502+106$. The red circles and the darkgreen bowtie at GeV energies represent the data of the outburst state of 8 August 2008; the red bowtie represents the Fermi LAT outburst spectrum averaged over the days 5-10 August and the downward pointing arrow is the INTEGRAL IBIS/ISGRI upper limit of 9-11 August. The blue circles represent the post-flare state of 15-22 August 2008 (Swift XRT and UVOT) and the blue bowtie is the spectrum in the low gamma-ray state observed by Fermi during its first 3 months in orbit (Abdo et al. 2009), equivalent to the Fermi LAT post-flare state after 11 August 2008 (see Abdo et al. 2010b). The NOT data (red open squares) refer to 13 August 2008. The ASCA and CGRO EGRET data (blue) and the grey open circles represent a historical quiescent radio-to-gamma-ray state. The optical/UV data have been corrected for dust extinction in our Galaxy using $E_{B-V}=0.032$ (Schlegel et al. 1998) and the extinction curve of Cardelli et al. (1989). The optical magnitudes from NOT and the optical-UV magnitudes from UVOT have been converted to fluxes according to Fukugita et al. (1995) and to Poole et al. (2008), respectively. The errors include the statistical and systematic uncertainties. The solid curves represent the synchrotron plus synchrotron self-Compton and external Compton model we used to fit the data of the highest gamma-ray state (light green), average outburst gamma-ray state (orange), the low state (cyan) and the historical quiescent state (black). See text and Table 2 for the model parameters. The black dashed curve represents the thermal emission from the dust torus, the accretion disk and its corona. The models do not account for the radio emission, that is likely produced in larger regions than the homogeneous one where the high energy emission takes place.

flux measurements of the Seyfert galaxy, to compare its longand short-term variability in X-ray and optical. Significant X-ray variability of up to $\sim 60 \%$ was detected on a timescale of 10 days and of $\sim 50 \%$ in almost 2 years. The $V$-band variability is less pronounced on both timescales, with an amplitude of $\sim 15-20 \%$. The light curves in the other UVOT filters have a behavior similar to the $V$-band, with somewhat larger variability amplitude, although the UV filter data have larger errors than the optical ones. We note that the long-term X-ray and optical variations are correlated (Fig. 2b).
The XRT spectrum of the blazar on 8 August 2008 is satisfactorily ( $\chi^{2}=32.5$ for 28 d.o.f.) fitted by a single power-law $F_{E} \propto E^{-\Gamma}$ of photon index $\Gamma=1.52 \pm 0.09$ (errors in the fitted $\mathrm{X}$-ray spectral parameters are at $90 \%$ confidence) and the flux in $2-10 \mathrm{keV}$ is $1.8 \times 10^{-12} \mathrm{erg} \mathrm{s}^{-1} \mathrm{~cm}^{-2}$. These results are consistent with those of Abdo et al. (2010b), who integrated the X-ray spectral signal over four days around the outburst. The extrapolation of the XRT flux to the IBIS energy range of 15-60 keV yields a flux of $3.8 \times 10^{-12} \mathrm{erg} \mathrm{s}^{-1} \mathrm{~cm}^{-2}$, which is six times lower than the flux measured by IBIS for the detected source. This 
independently suggests that, barring an improbably large spectral change between the soft and hard X-ray ranges, the IBIS source does not coincide with the blazar.

A single power-law describes well $\left(\chi^{2}=46.3\right.$ for 52 d.o.f.) the spectrum of Mkn 841 of 8 August 2008. The photon in$\operatorname{dex}$ is $\Gamma=2.03 \pm 0.07$ and the fitted flux in $2-10 \mathrm{keV}$ is $1.2 \times 10^{-11} \mathrm{erg} \mathrm{s}^{-1} \mathrm{~cm}^{-2}$. The extrapolation to the IBIS energy range yields a flux of $9.9 \times 10^{-12} \mathrm{erg} \mathrm{s}^{-1} \mathrm{~cm}^{-2}(15-60 \mathrm{keV})$, which is 2.4 times lower than measured by IBIS, and therefore compatible with that measurement, both taking into account the slight lack of simultaneity between the XRT spectrum and IBIS detection and assuming a spectral change between soft and hard $\mathrm{X}$-ray range caused by the emergence of a reflected Compton hump (see Discussion). No significant X-ray spectral variability is seen during the August 2008 campaign. Comparing our XRT spectrum of Mkn 841 with those of previous epochs reported by Grupe et al. (2010), we find some modest, marginally significant X-ray spectral variability, correlated with the flux in the sense of a harder spectrum being found in lower X-ray emission state. The broad-band UVOT spectra indicate a rather constant UV-optical spectral slope.

\subsection{Nordic Optical Telescope}

The results of the NOT observations are reported in Table 1. For the photometry of Mkn 841 , we used a rather small aperture (5-pixel radius equivalent to $0.95 \mathrm{arcsec}$ ) in order to reduce the host galaxy contribution. However, we noted that the profile of one of the comparison star and the target are very similar in the $B$ - and $V$-band images, leading us to conclude that the host galaxy contribution is negligible. The NOT measurement in $V$-band of Mkn 841 is consistent with the INTEGRAL OMC measurement within the errors, taking into account the difference in filter central wavelength.

\section{Discussion}

\subsection{PKS $1502+106$}

The data of our multiwavelength campaign in August 2008 are reported in Fig. 3, together with archival non-simultaneous data that sample a quiescent broad-band spectral state. We also show the Fermi LAT spectrum detected during the first three months of the survey (Abdo et al. 2009). Abdo et al. (2010b) modeled the spectral energy distribution of PKS $1502+106$ with a synchrotron component at radio-to-UV wavelengths and inverse Compton scattering at higher energies. In particular, synchrotron self-Compton radiation is mostly responsible for the X-ray spectrum, while external Compton upscattering is invoked to reproduce the MeV-GeV spectrum that has a curved shape in the lower state and a power-law slope during the flare (5-10 August 2010).

To model both the August 2008 low and high states and the archival spectral energy distributions, we applied a simple, one-zone, homogeneous leptonic model based on synchrotron radiation at radio-to-UV frequencies, and inverse Compton upscattering of both synchrotron photons (self-Compton) and external photons of the broad emission-line region (BLR), dominating at X-ray and gamma-ray frequencies, respectively, following an approach similar to that of Abdo et al. (2010b). The details of the model are described in Ghisellini et al. (2009) and Ghisellini et al. (2010). A strong constraint of the model is the variation in the $\mathrm{MeV}-\mathrm{GeV}$ spectrum in August 2008, indicating that the peak frequency of the external Compton component changes significantly between low and outburst state. We note that the INTEGRAL IBIS upper limit also provides a stringent constraint. Although the optical-UV spectrum follows a steep single power-law and does not require any additional thermal component (Fig. 3), the presence of an external Compton component at gamma-ray frequencies appears to point to a powering source of the BLR. We identify this with an accretion disk whose luminosity is limited by the smooth shape of the optical-UV spectrum to be no higher than $L_{\mathrm{d}} \simeq 1.7 \times 10^{46} \mathrm{erg} \mathrm{s}^{-1}$, consistent with that postulated by Abdo et al. (2010b). This is $\sim 11 \%$ of the Eddington luminosity for an assumed central black hole mass of $\sim 10^{9} M_{\odot}$, therefore in this blazar the jet power exceeds by a large amount the accretion power (see Ghisellini et al. 2010). The radius of the BLR is assumed to be $R_{\mathrm{BLR}}=4.1 \times 10^{17} \mathrm{~cm}$. The total X-ray corona luminosity is assumed to be $30 \%$ of $L_{\mathrm{d}}$, and its spectral shape $\propto v^{-1} \exp (-h v / 150 \mathrm{keV})$. The model parameters for the present spectral energy distributions are reported in Table 2 and the corresponding model curves are overplotted on the data in Fig. 3 in the same colors indicated in the table. The jet viewing angle $\theta_{\mathrm{v}}$ is $2.3^{\circ}$ for all states.

Our spectral energy distribution analysis differs from that of Abdo et al. (2010b) in the changes of the parameters that are responsible for variability among the various emission states (see Table 2). At variance with Abdo et al. (2010b), the parameters that exhibit the largest variations in our modeling are: (1) the magnetic field $B$, that increases by an order of magnitude between the highest and lowest gamma-ray state; (2) the total power injected in the jet $P_{\mathrm{i}}^{\prime}$, which is a factor 10 higher in the highest than in the lowest gamma-ray state; and (3) the random Lorentz factor $\gamma_{\mathrm{b}}$ of the electron distribution cooling break, which is significantly larger in the highest gamma-ray state, consistent with the harder spectrum. The variation in the magnetic field is consistent with the dominance of the inverse Compton cooling in the brightest gamma-ray state of 8 August 2008: the higher intensity of the inverse Compton scattering radiation implies that the ratio of the radiation energy density to the magnetic field energy density is relatively large, while the magnetic energy density dominates this ratio in lower inverse Compton states. The increase in the injected power $P_{\mathrm{i}}^{\prime}$ is related to an enhancement in the activity of the acceleration mechanism, so that the observed correlation between $P_{\mathrm{i}}^{\prime}$ and $\gamma_{\mathrm{b}}$ is not unexpected. We also find that the shape of the particle distribution changes between the low and hard states, with harder slopes below $\gamma_{\mathrm{b}}$ and steeper ones above. The synchrotron component, traced by the optical data, does not vary as dramatically as the inverse Compton component $(\mathrm{MeV}-\mathrm{GeV})$ on the same timescale, a behavior seen, for instance, in the blazars 3C 279 (see Wehrle et al. 1998 and Giuliani et al. 2009) and PKS 1454 354 (Ghisellini et al. 2009), but different from that of other wellmonitored blazar sources such as 3C 454.3 (Bonning et al. 2009; Vercellone et al. 2009; Donnarumma et al. 2009; Vercellone et al. 2010; Pacciani et al. 2010) and PKS 1510-089 (Abdo et al. 2010e; D'Ammando et al. 2011), where the correlated variations in optical and gamma-rays have comparable amplitudes on timescales from days to years (see however Ghisellini et al. 2007).

A multiwavelength monitoring of a few bright Fermi blazar sources on a range of timescales from days to a few years would allow us to obtain sensitive insight into the undoubtedly complex jet behavior. If used systematically in these campaigns, INTEGRAL will measure, or place constraining limits on, the flux at hard X-ray energies, that is crucial for precisely locating the peak of the high energy component. Besides this intrinsic merit, INTEGRAL IBIS - with its large field-of-view - often allows serendipitous detections of sources located within a few degrees of the primary target. Both in the present case, with the 
Table 2. Parameters of the theoretical spectral energy distributions of PKS 1502+106.

\begin{tabular}{llllllllllllll}
\hline \hline $\begin{array}{l}\text { date }^{a} \\
(1)\end{array}$ & $\begin{array}{l}R_{\mathrm{diss}}{ }^{b} \\
(2)\end{array}$ & $\begin{array}{l}P_{\mathrm{i}}^{\prime, c} \\
(3)\end{array}$ & $\begin{array}{l}B^{d} \\
4)\end{array}$ & $\begin{array}{l}\Gamma^{e} \\
(5)\end{array}$ & $\begin{array}{l}\gamma_{\mathrm{b}}^{f} \\
(6)\end{array}$ & $\begin{array}{l}\gamma_{\max }^{g} \\
(7)\end{array}$ & $\begin{array}{l}s_{1}^{h} \\
(8)\end{array}$ & $\begin{array}{l}s_{2}^{i} \\
(9)\end{array}$ & $\begin{array}{l}\log P_{\mathrm{r}}^{j} \\
(10)\end{array}$ & $\begin{array}{l}\log P_{\mathrm{B}}^{k} \\
(11)\end{array}$ & $\begin{array}{l}\log P_{\mathrm{e}}^{l} \\
(12)\end{array}$ & $\begin{array}{l}\log P_{\mathrm{p}}^{m} \\
(13)\end{array}$ \\
\hline Fermi LAT, 8 Aug. 2008 (light green) & $300(1000)$ & 0.16 & 0.75 & 19 & 3000 & 6000 & -1 & 2.6 & 46.8 & 44.8 & 44.9 & 47.2 \\
Fermi LAT, 5-10 Aug. 2008 (orange) & $195(650)$ & 0.1 & 2.2 & 19 & 500 & 5000 & -1 & 2.4 & 47.1 & 45.4 & 45.0 & 47.2 \\
15-22 Aug. 2008 (cyan) & $210(700)$ & 0.05 & 2.2 & 19 & 250 & 4300 & -1 & 2.3 & 46.3 & 45.5 & 44.8 & 47.1 \\
historical (black) & $120(400)$ & 0.015 & 7.3 & 10 & 800 & 2000 & 0.25 & 2.2 & 45.1 & 45.5 & 45.5 & 45.9 \\
\hline
\end{tabular}

Notes. ${ }^{(a)}$ epoch/state (colors refer to the model curves in Fig. 3); ${ }^{(b)}$ dissipation radius in units of $10^{15} \mathrm{~cm}$ and (in parenthesis) in units of gravitational radii; ${ }^{(c)}$ power injected in the blob calculated in the comoving frame, in units of $10^{45} \mathrm{erg} \mathrm{s}^{-1}$; ${ }^{(d)}$ magnetic field, in gauss; ${ }^{(e)}$ bulk Lorentz factor at $R_{\text {diss }} ;{ }^{(f)}$ break random Lorentz factors of the injected electrons; ${ }^{(g)}$ maximum random Lorentz factors of the injected electrons; ${ }^{(h)}$ slope of the injected electron distribution $[Q(\gamma)]$ below $\gamma_{\mathrm{b}} ;{ }^{(i)}$ slope of the injected electron distribution $[Q(\gamma)]$ above $\gamma_{\mathrm{b}} ;{ }^{(j)}$ Logarithm of the jet power in the form of radiation, in $\mathrm{erg} \mathrm{s}^{-1}$; ${ }^{(k)}$ Logarithm of Poynting flux, in erg s${ }^{-1}$; ${ }^{(l)}$ Logarithm of bulk motion power of electrons, in erg s ${ }^{-1}$; ${ }^{(m)}$ Logarithm of bulk motion power of protons, in $\mathrm{erg} \mathrm{s}^{-1}$.

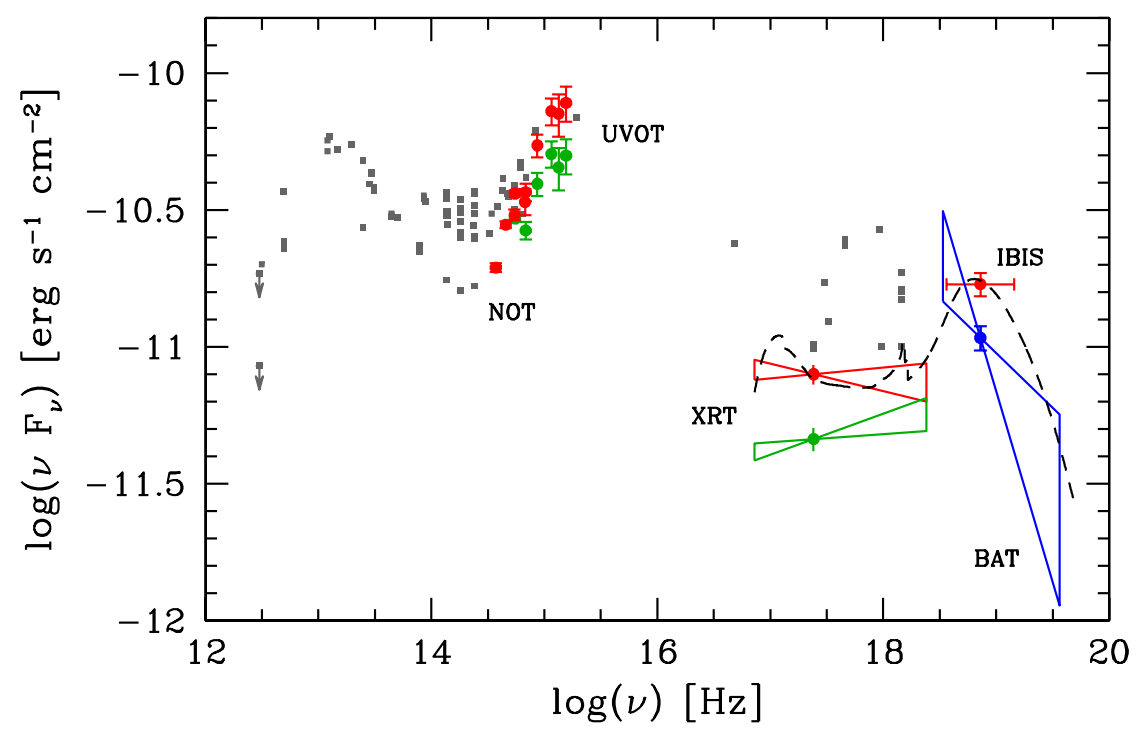

Fig. 4. Spectral energy distributions of Mkn 841 in August 2008 (red symbols: INTEGRAL IBIS/ISGRI, 9-11 August; Swift XRT spectrum, 8 August; Swift UVOT, 13-15 August; NOT photometry, 13 August), on 3 February 2007 (green symbols: Swift XRT and UVOT) and at prior epochs, not from simultaneous data (taken from the NED Extragalactic Database, grey squares). The optical/UV data have been corrected for dust extinction in our Galaxy using $E_{B-V}=0.03$ (Schlegel et al. 1998) and the extinction curve of Cardelli et al. (1989). The optical magnitudes from NOT and the optical-UV magnitudes from UVOT have been converted to fluxes according to Fukugita et al. (1995) and to Poole et al. (2008), respectively. In addition, the Swift BAT spectrum from the first 39 months of the mission is shown in blue (December 2004-February 2008, Cusumano et al. 2010). The errors include the statistical and systematic uncertainties. The model fitted by Bianchi et al. (2001) to the 1999 BeppoSAX spectrum, including a soft X-ray excess, a Comptonized spectrum of a hot corona with a high energy cutoff and a reflected component, is shown as a dashed curve. detection of the Seyfert Mkn 841, and in other previous circumstances (e.g., Pian et al. 2005), this advantage has led to an additional contribution to the study of the hard X-ray extragalactic sources.

\section{2. $M k n 841$}

BeppoSAX observations of the Seyfert 1 Mkn 841 in 1999 and simultaneous XMM-Newton and BeppoSAX observations in 2001 showed a high energy cut-off at around $100 \mathrm{keV}$ (Bianchi et al. 2001; Petrucci et al. 2002; Bianchi et al. 2004). As noted by Ubertini et al. (2008), this strongly suggests that this source, despite being 20 times brighter in X-rays than PKS 1502+106 when quiescent, is unlikely to emit in the Fermi LAT domain at the reported level (Abdo et al. 2010b). Therefore, the Fermi LAT detection can be firmly identified with emission from the blazar. On the other hand, the imaging and spectral arguments made in the previous section suggest that the hard X-rays detected by IBIS should be entirely attributed to the Seyfert.

From the January 2006 and present INTEGRAL IBIS observations, there is no evidence of significant variability in the hard X-ray flux of Mkn 841. This source was first detected in hard X-rays (50-150 keV) in 1996 by CGRO OSSE (Zdziarski et al. 2000) at a flux level of $(6 \pm 2) \times 10^{-11} \mathrm{erg} \mathrm{s}^{-1} \mathrm{~cm}^{-2}$, an order of magnitude higher than the extrapolation of the IBIS flux in the OSSE band using a reflected Compton spectrum (see below and Fig. 4), a difference that is not to be considered dramatic owing to the low significance of the OSSE detection (we tend to exclude contamination of the OSSE detection by PKS 1502+106, see below). On the other hand, the Swift BAT detection of Mkn 841 obtained during the first 39 months of the mission (Cusumano et al. 2010) is somewhat lower than the IBIS flux (see Fig. 4). This indicates a moderate secular variability in the hard X-ray emission. The softer $(0.3-10 \mathrm{keV}) \mathrm{X}$-ray flux varies more substantially during the Swift mission lifetime, and exhibits maximum amplitude variability of about $\sim 50 \%$ on both month and day timescales (Fig. 2), in agreement with the behavior observed by other satellites, which detected even faster variations (George et al. 1993; Nandra et al. 1995; Bianchi et al. 2001; Petrucci et al. 2007).

In Fig. 4 we compare the spectral energy distribution of Mkn 841 based on the INTEGRAL, Swift, and NOT quasisimultaneous (within few days) observations of August 2008 with historical data and the Swift XRT and UVOT observations of February 2007. The Swift BAT average flux and spectrum from the 39-month survey (Cusumano et al. 2010) are also shown. The spectrum has the overall typical shape of a Seyfert 1 nucleus, with a prominent rising optical/UV component, a relatively soft spectrum in the $0.3-10 \mathrm{keV}$ range and a hard X-ray component with a cutoff around $150 \mathrm{keV}$, which suggests a relatively high coronal maximum temperature. The NOT, UVOT, and XRT measurements of August 2008 indicate a relatively high state. The limited accuracy of XRT does not allow us to determine whether there is a soft X-ray excess, which is often observed in Seyferts and was first detected precisely in this 
object (Arnaud et al. 1985). However, as already noted, the lower state of February 2007 is also harder, suggesting a variable soft component, more prominent in the high states. The correlation between X-ray spectral index and flux (harder X-ray spectrum during lower X-ray states) is confirmed by all Swift XRT observations of this source (see Grupe et al. 2010).

We superimposed on the data, without any scaling, the model of a black body of characteristic temperature $\sim 100 \mathrm{eV}$ to reproduce phenomenologically the soft X-ray excess, a power-law corresponding to the Compton upscattering of the coronal soft $\mathrm{X}$-rays, with a cutoff at $100 \mathrm{keV}$, and reflected continuum and fluorescent iron line component at hard X-rays, which was proposed by Bianchi et al. (2001) as the most accurate description of the 1999 BeppoSAX spectrum (see also Ponti et al. 2008, for a Suzaku observation of the hard X-ray spectrum). Apart from the iron line, which is beyond reach of the limited sensitivity and spectral resolution of XRT, the model satisfactorily reproduces the XRT, IBIS, and BAT data (Fig. 4).

The average optical-to-X-ray color index $\alpha_{\text {ox }}$ obtained from the present and previous (Grupe et al. 2010) XRT and UVOT observations of Mkn 841 is consistent with the relationship between $\alpha_{\mathrm{Ox}}$ and UV luminosity found in optically selected AGNs (Strateva et al. 2005; Steffen et al. 2006), although this is not the case when the $\alpha_{\text {ox }}$ values of the individual Swift observations are compared, because the X-ray variations are larger than the optical ones. Correlated X-ray and optical variability with larger amplitude in X-rays than optical has been seen in other Seyferts (e.g., Breedt et al. 2010), although the opposite is also seen in objects of this AGN class. This optical-X-ray variability of Mkn 841 and its correlated X-ray flux and spectral variations (steeper spectrum for brighter states) must both be taken into account when constructing an emission scenario for this source. However, our data are inadequate to provide a detailed physical characterization of the soft X-ray excess component and its relation to the optical-UV bump. This requires a complex model (see e.g. Petrucci et al. 2007; Ponti et al. 2008; Longinotti et al. 2010) and an accurate and sensitive simultaneous monitoring in optical/UV and X-rays.

We conclude with a note about the possible contamination of the hard X-ray flux of Mkn 841 in the observations that preceded the INTEGRAL one: the model we used to reproduce the broad-band spectrum of PKS $1502+106$ (Fig. 3) predicts an intensity of about $5 \times 10^{-12} \mathrm{erg} \mathrm{s}^{-1} \mathrm{~cm}^{-2}$ in hard X-rays, with little variability among very different $\mathrm{MeV}-\mathrm{GeV}$ emission states. This is a rather negligible fraction (20\%) of the IBIS detected flux. Assuming therefore that neither the blazar nor the Seyfert vary dramatically in hard X-rays, both the BeppoSAX PDS measurement of Mkn 841 (Bianchi et al. 2001; Petrucci et al. 2002), which is consistent with IBIS, and the CGRO OSSE measurement (Johnson et al. 2000) do not contain significant contributions from the blazar, which lies in the field of view of the two instruments during Mkn 841 observations. The uncertainty circle of the Swift BAT Mkn 841 detection has a radius of 2.5 arcmin (Cusumano et al. 2010), which excludes contamination from PKS 1502+106.

Acknowledgements. We thank C. Winkler, P. Kretschmar and C. Sanchez for assistance with the INTEGRAL observations scheduling, and P. Lubinski and the INTEGRAL Science Data Center staff for help with data reduction. We benefitted from financial support through contracts ASI-INAF I/023/05/0 and ASI-INAF I/088/06/0. R.H. acknowleges support from ESA PECS 98023 and by the GA CR 102/09/0997 and 205/08/1227. This research has made use of NASA's Astrophysics Data System Bibliographic Services, of the SIMBAD Astronomical Database, and of the NASA/IPAC Extragalactic Database (NED), which is operated by the Jet Propulsion Laboratory, California Institute of Technology, under contract with the National Aeronautics and Space Administration.

\section{References}

Abdo, A. A., Ackermann, M., Ajello, M., et al. 2009, ApJ, 700, 597 Abdo, A. A., Ackermann, M., Agudo, I., et al. 2010a, ApJ, 716, 30 Abdo, A. A., Ackermann, M., Ajello, M., et al. 2010b, ApJ, 710, 810 Abdo, A. A., Ackermann, M., Ajello, M., et al. 2010c, ApJ, 710, 127 Abdo, A. A., Ackermann, M., Ajello, M., et al. 2010d, ApJ, 722, 520 Abdo, A. A., Ackermann, M., Agudo, I., et al. 2010e, ApJ, 721, 1425 An, T., Hong, X. Y., Venturi, T., Jiang, D. R., \& Wang, W. H. 2004, A\&A, 421, 839

Arnaud, K. A., Branduardi-Raymont, G., Culhane, J. L., et al. 1985, MNRAS, 217,105

Atwood, W. B., Abdo, A. A., Ackermann, M., et al. 2009, ApJ, 697, 1071

Beckmann, V., Soldi, S., Ricci, C., et al. 2009, A\&A, 505, 417

Bianchi, S., Matt, G., Haardt, F., et al. 2001, A\&A, 376, 77

Bianchi, S., Matt, G., Balestra, I., Guainazzi, M., \& Perola, G. C. 2004, A\&A 422, 65

Bird, A. J., Bazzano, A., Bassani, L., et al. 2010, ApJS, 186,

Blazek, M., \& Hudec, R. 2009, Proceedings of The Extreme sky: Sampling the Universe above $10 \mathrm{keV}$. October 13-17 2009, Otranto (Lecce) Italy. Published online at http://pos.sissa.it/cgi-bin/reader/ conf. cgi?confid=96, 15

Bonning, E. W., Bailyn, C., Urry, C. M., et al. 2009, ApJ, 697, L81

Böttcher, M., \& Dermer, C. D. 2010, ApJ, 711, 445

Breedt, E., McHardy, I. M., Arévalo, P., et al. 2010, MNRAS, 403, 605

Burrows, D. N., Hill, J. E., Nousek, J., et al. 2005, Space Sci. Rev., 120, 165

Cardelli, J. A., Clayton, G. C., \& Mathis, J. S. 1989, ApJ, 345, 245

Courvoisier, T. J.-L., Walter, R., Beckmann, V., et al. 2003, A\&A, 411, L53

Cusumano, G., La Parola, V., Segreto, A., et al. 2010, A\&A, 510, A48

D’Ammando, F., Raiteri, C. M., Villata, M., et al. 2011, A\&A, submitted

Donnarumma, I., Pucella, G., Vittorini, V., et al. 2009, ApJ, 707, 1115

Fukugita, M., Shimasaku, K., \& Ichikawa, T. 1995, PASP, 107, 945

George, I. M., Nandra, K., Fabian, A. C., et al. 1993, MNRAS, 260, 111

George, I. M., Nandra, K., Turner, T. J., \& Celotti, A. 1994, ApJ, 436, L59

Gehrels, N., Chincarini, G., Giommi, P., et al. 2004, ApJ, 611, 1005

Ghisellini, G., Foschini, L., Tavecchio, F., \& Pian, E. 2007, MNRAS, 382, L 82

Ghisellini, G., Tavecchio, F., \& Ghirlanda, G. 2009, MNRAS, 399, 2041

Ghisellini, G., Tavecchio, F., Foschini, L., et al. 2010, MNRAS, 402, 497

Giuliani, A., D'Ammando, F., Vercellone, S., et al. 2009, A\&A, 494, 509

Goldwurm, A., David, P., Foschini, L., et al., 2003, A\&A 411, L223

Grupe, D., Komossa, S., Leighly, K. M., \& Page, K. L. 2010, ApJS, 187, 64

Hartman, R. C., Bertsch, D. L., Bloom, S. D., et al. 1999, ApJS, 123, 79 Impey, C. D., \& Tapia, S. 1988, ApJ, 333, 666

Kalberla, P. M. W., Burton, W. B., Hartmann, D., et al. 2005, A\&A, 440, 775

Lahteenmaki, A., \& Valtaoja, E. 1999, ApJ, 521, 493

Lebrun, F., Leray, J. P., Lavocat, P., et al., 2003, A\&A, 411, L141

Lister, M. L., \& Homan, D. C. 2005, AJ, 130, 1389

Longinotti, A. L., Costantini, E., Petrucci, P.-O., et al. 2010, A\&A, 510, A92

Lund, N., Budtz-Jørgensen, G., Westergaard, N. J., et al. 2003, A\&A, 411, L231

Marscher, A. P., Jorstad, S. G., Larionov, V. M., et al. 2010, ApJ, 710, L131

Mas-Hesse, J. M., Giménez, A., Culhane, L., et al. 2003, A\&A, 411, L261

Nandra, K., Turner, T. J., George, I. M., et al. 1995, MNRAS, 273, 85

Pacciani, L. Vittorini, V., Tavani, M., et al. 2010, ApJ, 716, L170

Petrucci, P. O., Henri, G., Maraschi, L., et al. 2002, A\&A, 388, L5

Petrucci, P. O., Ponti, G., Matt, G., et al. 2007, A\&A, 470, 889

Pian, E., Foschini, L., Beckmann, V., et al. 2005, A\&A, 429, 427

Ponti, G., Petrucci, P. O., Matt, G., et al. 2008, SF2A-2008: Proceedings of the Annual meeting of the French Society of Astronomy and Astrophysics, ed. C. Charbonnel, F. Combes, \& R. Samadi. Available online at http://proc. sf2a.asso.fr, p.263

Poole, T. S., Breeveld, A. A., Page, M. J., et al. 2008, MNRAS, 383, 627

Roming, P. W. A., Kennedy, T. E., Mason, K. O., et al. 2005, Space Sci. Rev., 120,95

Schlegel, D. J., Finkbeiner, D. P., \& Davis, M. 1998, ApJ, 500, 525

Steffen, A. T., Strateva, I. V., Brandt, W. N., et al. 2006, AJ, 131, 2826

Strateva, I. V., Brandt, W. N., Schneider, D. P., Vanden Berk, D. G., \& Vignali, C. 2005, AJ, 130,387

Ubertini, P., Lebrun, F., Di Cocco, G., et al. 2003, A\&A, 411, L131

Ubertini, P., Bazzano, A., Bird, A. J., et al. 2008, The Astronomer's Telegram N. 1652

Vercellone, S., Chen, A. W., Vittorini, V., et al. 2009, ApJ, 690, 1018

Vercellone, S., D’Ammando, F., Vittorini, V., et al. 2010, ApJ, 712, 405

Wehrle, A. E., Pian, E., Urry, C. M., et al. 1998, ApJ, 497, 178

Westergaard, N.J, Kretschmar, P., Oxborrow, C. A., et al. 2003, A\&A, 411, L257

Winkler, C., Courvoisier, T. J.-L., Di Cocco, G., et al. 2003, A\&A, 411, L1

Zdziarski, A. A., Poutanen, J., \& Johnson, W. N. 2000, ApJ, 542, 703 